\title{
THE REALIZATION OF ABSTRACT STRATIFIED SETS
}

\author{
By Hiroko NATSUME
}

\section{§1. Introduction.}

It is well-known that $n$-dimensional differentiable manifolds can be realized in the $(2 n+1)$-dimensional Euclidean space.

Recently, R. Thom and J. Mather have introduced the notion of abstract stratified set, modelling after variety (manifold with singuralities). In this paper, we shall realize $n$-dimensional stratified sets in the $(2 n+1)$-dimensional Euclidean space.

DEFINITION 1 ([1]). A stratification $\mathcal{S}$ for a subset $V$ in $R^{N}$ is a locally finite family of pairwise disjoint submanifolds $X$ of $R^{N}$, satisfying the following conditions.

S1. Each $X \in \mathcal{S}$ lies in $V$, which is called a stratum of $\{V, \mathcal{S}\}$.

S2. Each point of $V$ is contained in the interior of some stratum.

S3. The frontier condition: for each stratum $X$, if a stratum $Y$ intersects with the closure $\bar{X}$ in $V$ of $X$, then $Y \subset \bar{X}$.

If $Y \subset \bar{X}, Y$ is said to be incident to $X$ and we write $Y<X$ or $X>Y$.

A topological space $V$ with a stratification $\mathcal{S}$ is called a stratified set. H. Whitney in [1] considered stratified sets with the following condition.

Whitney condition. For each pair of strata $(X, Y)$ such that $X>Y$, if both series of points $\left\{x_{i}\right\}$ in $X$ and $\left\{y_{i}\right\}$ in $Y$ converge to a point $y$ in $Y$ and the line through $x_{2}$ and $y_{2}$ converges to some line $l$ and the tangent space of $X$ at $x_{\imath}$ converges to some plane $P$, then $l \subset P$.

R. Thom ([3]) and J. Mather ([2]), axiomatizing stratified set together with a tubular neighbourhood system, introduced the following notion of abstract stratified sets.

Definition 2 ([2]). Let $V$ be a subset of $R^{N}$ with a stratification $\mathcal{S}$. A family $\mathscr{I}=\left\{\left(T_{X}, \pi_{X}, \rho_{X}\right)\right\}_{X \in S}$ is called a tubular neighbourhood system for $\mathcal{S}$, if it satisfies the following conditions:

T1. $T_{X}$ is an open neighbourhood of $X$ in $R^{N}$.

T2. $\pi_{X}: T_{X} \rightarrow X$ is a bundle such that there exists a bundle isomorphism $\varphi_{X}: T_{X} \rightarrow B_{X}$ where $B_{X}$ is the open unit ball bundle in an inner product bundle over $X$.

Received September 25, 1978 
T3. $\rho_{X}: T_{X} \rightarrow[0,1)$ is the function defined by $\rho_{X}(v)=\left\|\varphi_{X}(v)\right\|$ for $v \in T_{X}$.

T4. For $Y<X, \rho_{Y} \circ \pi_{X}(v)=\rho_{Y}(v)$ for $v \in T_{X} \cap T_{Y}$.

DEFINITION 3. Let $V$ be a subset of $R^{N}$ with a stratification $\mathcal{S}$. A family $\mathscr{I}=\left\{\left(T_{X}, \pi_{X}, \rho_{X}\right)\right\}_{X \in \mathcal{S}}$ is called a closed tubular neighbourhood system, if $T_{X}$ is a closed tubular neighbourhood of $X$ in $R^{N}$ and there exists a tubular neighbourhood system $\left.\mathscr{I}^{\prime}=\left\{T_{X}^{\prime}, \pi_{X}^{\prime}, \rho_{X}^{\prime}\right)\right\}_{X \in \mathcal{S}}$ for $\mathcal{S}$ such that $T_{X}^{\prime} \supset T_{X}, \pi_{X}^{\prime} \mid T_{X}$ $=\pi_{X}$ and $\rho_{X}^{\prime} \mid T_{X}=\rho_{X}$ for any $X$ in $\mathcal{S}$.

Definition 4 ([2]). An abstract stratified set is a triple $\{V, \mathcal{S}, \mathscr{I}\}$ satisfying the following conditions.

A1. $V$ is a Hausdorff, locally compact topological space with a countable basis.

A2. $\mathcal{S}$ is a family of locally closed subsets of $V$ such that $V$ is the disjoint union of members of $\mathcal{S}$.

A3. Each stratum of $\{V, \mathcal{S}, \mathcal{I}\}$ is a smooth mainfold.

A4. The family $\mathcal{S}$ is locally finite.

A5. The frontier condition: the same as S3 in Definition 1.

If $Y \subset \bar{X}, Y$ is also said to be incident to $X$ and we write $Y<X$ or $X>Y$. A6. $I$ is a family of triples $\left\{\left(T_{X}, \pi_{X}, \rho_{X}\right)\right\}_{X \in \mathcal{S}}$, where $T_{X}$ is a closed neighbourhood of $X$ in $V$ such that there exists an open neighbourhood $T_{X}^{\prime} \supset T_{X}$ of $X$ in $V, \pi_{X}$ is a continuous retraction of $T_{X}^{\prime}$ to $X$ and $\rho_{X}$ is a non-negative continuous function of $T_{X}^{\prime}$.

A7. $X=\rho_{X}^{-1}(0)$.

A8. If $X>Y$, then the mapping $\pi_{Y, X} \times \rho_{Y, X}: T_{Y, X} \rightarrow Y \times(0, \infty)$ is a smooth submersion, where $T_{Y, X}=T_{Y}^{\prime} \cap X, \pi_{Y, X}=\pi_{Y}\left|T_{Y, X}, \rho_{Y, X}=\rho_{Y}\right| T_{Y, X}$ and the inverse image of $Y \times(0,1]$ by $\pi_{Y, X} \times \rho_{Y, X}$ is equal to $T_{Y} \cap X$.

A9. For arbitrary strata $X, Y$ and $Z$, we have $\pi_{Z, Y} \circ \pi_{Y, X}=\pi_{Z, X}$ and $\rho_{Z, Y} \circ \pi_{Y, X}$ $=\rho_{Z, X}$ whenever both sides of these equations are defined.

Definition 5. We say that a stratified set $\{V, \mathcal{S}, \mathscr{I}\}$ is equivalent to $\left\{V^{\prime}, \mathcal{S}^{\prime}, \mathscr{I}^{\prime}\right\}$ if the following conditions hold.

(1) $V=V^{\prime}, \mathcal{S}=\mathcal{S}^{\prime}$ and for each stratum $X$ of $\mathcal{S}=\mathcal{S}^{\prime}$, the two smoothness structures on $X$ are the same.

(2) If $\mathscr{I}=\left\{\left(T_{X}, \pi_{X}, \rho_{X}\right)\right\}$ and $\mathscr{I}^{\prime}=\left\{\left(T_{X}^{\prime}, \pi_{X}^{\prime}, \rho_{X}^{\prime}\right)\right\}$, then for each stratum $X$ of $\mathcal{S}$, there exists a neighbourhood $T_{X}^{\prime \prime}$ of $X$ in $T_{X} \cap T_{X}^{\prime}$ such that $\rho_{X}\left|T_{X}^{\prime \prime}=\rho_{X}^{\prime}\right| T_{X}^{\prime \prime}$ and $\pi_{X}\left|T_{X}^{\prime \prime}=\pi_{X}^{\prime}\right| T_{X}^{\prime \prime}$.

Now, we introduce the notion of the relization of abstract stratified sets as follows:

Definition 6. An abstract stratified set $\{V, \mathcal{S}, \mathcal{I}\}$ is called to be realized in the Euclidean space $R^{N}$ if there is a homeomorphism $F: V \rightarrow R^{N}$ and an equivalent abstract stratified set $\left\{V, \mathcal{S}, \mathscr{I}^{\prime}\right\}$ to $\{V, \mathcal{S}, \mathscr{T}\}$ satisfying the following conditions :

(1) $V_{1}=F(V)$ is a stratified set with a stratification $\mathcal{S}_{1}=\{F(X) ; X \in \mathcal{S}\}$ and a 
closed tubular neighbourhood system

$$
\mathscr{I}_{1}=\left\{\left(T_{F(X)}, \pi_{F(X)}, \rho_{F(X)}\right)\right\} .
$$

(2) For each triple $\left(T_{X}, \pi_{X}, \rho_{X}\right) \in \mathscr{I}^{\prime}, F$ is a diffeomorphism of $T_{X}$ into $T_{F(X)}$, compatible with the retractions $\pi_{X}$ and $\pi_{F(X)}$, that is, $F \circ \pi_{X}=\pi_{F(X)} \circ F$.

(3) $\rho_{X}=\rho_{F(X)} \circ F$.

$F$ is said to be a realization of $\{V, \mathcal{S}, \mathscr{I}\}$ in $R^{N}$.

Definition 7. Two realizations $F_{0}$ and $F_{1}$ of $\{V, \mathcal{S}, \mathscr{I}\}$ in $R^{N}$ are called isotopic if there exists a realization $H$ of $\{V, \mathcal{S}, \mathscr{I}\} \times I$ in $R^{N+1}$ which satisfies the following conditions:

(1) $H(x, t)=\left(H_{t}(x), t\right)$ where $H_{t}$ is a realization of $\{V, \mathcal{S}, \mathscr{I}\}$ in $R^{N}$ for each $t \in[0,1]$.

(2) $H_{0}=F_{0}$ and $H_{1}=F_{1}$.

Remark. We can define naturally a stratification on $\{V, \mathcal{S}, \mathscr{I}\} \times I$ with a tubular neighbourhood system ([3]).

Our main result is the following.

THEOREM. Every paracompact abstract stratıfied set $\{V, \mathcal{S}, \mathscr{I}\}$ with $\operatorname{dim} V=n$ can be realized in $R^{2 n+1}$ such that the image of the realization is the stratified set satisfying the Whitney condition. All realizations of $\{V, \mathcal{S}, \mathcal{I}\}$ in $R^{N}$ are isotopic if $N \geqq 2 n+2$.

Remark 1. The dimension of $V$ is the topological dimension.

Remark 2. More generally, every continuous mapping $F$ from an $n$ dimensional abstract stratified set $\{V, \mathcal{S}, \mathscr{I}\}$ into $R^{2 n+1}$ can be approximated by a realization.

\section{$\S 2$. Preliminary lemmas.}

In this section, we prove several lemmas for the proof of the theorem.

LEMMA 1. Let $\pi: E \rightarrow M$ be a fibre bundle with a compact manifold $F$ as the fibre, $h$ a diffeomorphism of $M$ onto $M^{\prime}, S$ a sphere bundle of $M^{\prime}$ and $p$ the projection of $S$ to $M^{\prime}$. Suppose that $\operatorname{dim} S \geqq 2 \operatorname{dim} E+2$, then we have an embedding $\tilde{h}$ of $E$ into $S$ such that $h \circ \pi=p \circ \tilde{h}$. Moreover, such an embedding $\tilde{h}$ can be obtained by extending any given embedding $\tilde{h}_{0}: \pi^{-1}(A) \rightarrow p^{-1}(h(A))$ for a closed set $A$ in $M$ with $h \circ \pi=p \circ \tilde{h}_{0}$.

Proof. Let $A$ be a closed set in $M$ and $\tilde{h}_{0}$ a given embedding defined on $\pi^{-1}(A)$ into $p^{-1}(h(A))$ with $h \circ \pi=p \circ \tilde{h}_{0}$. Let $U$ be an open set in $M$, and $V$ a compact set with $V \subset U$ such that both fibre bundles $\pi$ and $p$ are trivialized in $U$ and $h(U)$. Then, it is sufficient to prove that $\tilde{h}_{0}$ can be extended to an 
embedding $\tilde{h}$ of a closed set $\pi^{-1}(A \cup V)$. Let $\varphi$ be a trivialization $\pi^{-1}(U) \cong U \times F$ and $\phi$ a trivialization $p^{-1}(h(U)) \cong h(U) \times S^{n}$ with $n=\operatorname{dim} S-\operatorname{dim} M$. Define $\tilde{k}_{0}:(A \cap U) \times F \rightarrow S^{n}$ by $\tilde{k}_{0}=p^{\prime} \circ \phi^{\circ} h_{0} \circ \varphi^{-1}$ where $p^{\prime}$ is a natural projection from $h(U) \times S^{n}$ to $S^{n}$. Then $\tilde{k}_{0}$ can be extended to a smooth map $\tilde{k}_{1}$ on $U \times F$ because $\pi_{\jmath}\left(S^{n}\right)=0$ for any $\jmath<\operatorname{dim} E$. Moreover Thom's transversality theorem ([4]) assures that $\tilde{k}_{1}$ can be approximated by a mapping $\tilde{k}$ with $\tilde{k}=\tilde{k}_{1}$ on $(A \cap U) \times F$ and satisfying the following conditions:

(1) The cross-section $\jmath_{1} \tilde{k}$ is transverse to $\Sigma$ where $\Sigma$ is the subset in $J^{1}\left(U \times F, S^{n}\right)$ consisting of the first jets of those mappings $g$ which are of full rank as mappings of $F$ into $S^{n}$ by fixing points of $U$.

(2) The mapping $\jmath_{0} \tilde{k} \times j_{0} \tilde{k}$ is transeverse to $\Sigma_{1}$ where $\Sigma_{1}$ is the subset in $J^{0}\left(U \times F, S^{n}\right) \times J^{0}\left(U \times F, S^{n}\right)$ consisting of all elements of the form $((u, f, s)$, $\left.\left(u, f^{\prime}, s\right)\right)$.

As easy calculation shows, the transversality in the conditions (1) or (2) means the disjointness of $\jmath_{1} \tilde{k}(U \times F)$ from $\Sigma$ or $\jmath_{0} \tilde{k}(U \times F) \times \jmath_{0} \tilde{k}(U \times F)$ from $\Sigma_{1}$. Now, the desired embedding is obtained by defining $\tilde{h}=\tilde{h}_{0}$ on $\pi^{-1}(A)$ and $\tilde{h}=\phi^{-1} \circ(h \times \tilde{k}) \circ \varphi$ on $\pi^{-1}(V)$ with $(h \times \tilde{k})(u, f)=(h(u), \tilde{k}(u, f))$. This completes the proof of Lemma 1.

We introduce the following two conditions for convenience.

Definition 8. Let $X, Y$ and $Z$ be strata with $X>Y>Z, \xi_{Y}$ a vector field on $T_{Z} \cap Y$ and $\xi_{X}$ a vector field on $T_{Z} \cap X$. We say that $\xi_{X}$ and $\xi_{Y}$ are $\pi_{Y, X^{-}}$ related or $\xi_{X}$ is $\pi_{Y, X}$-related to $\xi_{Y}$ if $\left(\pi_{Y, X}\right)_{*} \xi_{X}=\xi_{Y}$.

Now, let $T_{X}(t)$ denote the subset $\rho_{X}^{-1}(t)$ of $T_{X}$. Let $X, Y$ and $Z$ be strata with $X>Y>Z, \xi$ a vector field on $T_{Z} \cap X$ and $\left\{\sigma_{t}\right\}$ one parameter family of local transformations defined by $\xi$. Suppose that for each point $x$ in $T_{Z} \cap X$ there is a point $x_{0}$ in $T_{z}(1) \cap X$ and a real number $t$ with $\sigma_{t}\left(x_{0}\right)=x$.

Definition 9. Under the above situation, a vector field $\eta$ on $T_{Z} \cap T_{Y} \cap X$ is said to be a sliding of a vector field $\eta_{0}$ on $T_{Z}(1) \cap T_{Y} \cap X$ along $\xi$ if $\eta\left(\sigma_{t}\left(x_{0}\right)\right)$ $=\sigma_{t *}\left(\eta_{0}\left(x_{0}\right)\right)$ for every $x_{0} \in T_{Z}(1)$ and $t \in[0, T]$.

As J. Mather has shown in [2], we can choose, for a given abstract stratified set, an equivalent abstract stratified set satisfying the following condition $\mathrm{A} 10$ and $\mathrm{A} 11$.

A10. If $X$ and $Y$ are strata and $T_{Y, X} \neq \Phi$, then $Y<X$.

A11. If $X$ and $Y$ are strata and $T_{X} \cap T_{Y} \neq \Phi$, then $X<Y, Y<X$, or $X=Y$.

LEMMA 2. Let $\{V, \mathcal{S}, \mathscr{I}\}$ be an abstract stratified set, satisfying A10 and A11. Then, there exists a family $\left\{\sigma_{t}^{X}\right\}$ of one parameter families of local transformations on $T_{X}$ for each stratum $X$ in $\mathcal{S}$ satisfying the following conditions :

(1) $\sigma_{t}^{Y}$ is smooth on $T_{Y} \cap X$ for each $X$ with $X>Y$.

(2) $\pi_{X} \circ \sigma_{t}^{X}(x)=\pi_{X}(x)$ for each $x$ in $T_{X}$.

(3) $\rho_{X} \circ \sigma_{t}^{X}(x)=\rho_{X}(x)-t$ for $x \in T_{X}$ and $t \in[0,1)$ whenever both sides of this equation are defined. 
(4) If $X>Y, \pi_{X} \circ \sigma_{t}^{Y}(x)=\sigma_{t}^{Y}\left(\pi_{X}(x)\right)$ for each $x$ in $T_{X} \cap T_{Y}$.

(5) If $X>Y, \rho_{Y} \circ \sigma_{t}^{X}(x)=\sigma_{Y}(x)$ and $\sigma_{s}^{Y} \circ \sigma_{t}^{X}(x)=\sigma_{t}^{X} \circ \sigma_{s}^{Y}(x)$ for any $x$ in $T_{X} \cap T_{Y}$.

Proof. It is sufficient to show that if the lemma is true for $\operatorname{dim} V \leqq k$, then it is true for $\operatorname{dim} V=k+1$. So we assume that $\operatorname{dim} V=k+1$. Let $\left\{\sigma_{t}^{Z}\right\}$ denote the one parameter family on $T_{Z} \cap V^{(k)}$ for each $Z$ in $V^{(k)}$ satisfying the conditions (1) (5). Here $V^{(k)}$ denotes the union of all strata $Y$ of $V$ with $\operatorname{dim} Y \leqq k$. Let $\eta_{Z, Y}$ be the vector field on $T_{Z} \cap Y$ which define $\left\{\sigma_{t}^{Z}\right\}$ on $T_{Z} \cap Y$. Let $X$ be an stratum in $\mathcal{S}$ with $\operatorname{dim} X=k+1$ and $\mathcal{S}_{X}$ the set of all strata $Y$ with $Y<X$. We define a smooth vector field $\eta_{Y, X}$ on $T_{Y} \cap X$ for each $Y \in \mathcal{S}_{X}$ satisfying the following conditions:

(a) $\eta_{Y, X}$ is tangential to the fibre of the projection $\pi_{Y, X}$.

(b) $\rho_{Y} \circ \sigma_{t}^{Y}(x)=\rho_{Y}(x)-t$ for each $x$ in $T_{Y} \cap X$ and $t \in[0,1)$.

(c) If $Z<Y<X, \eta_{Z, X}$ and $\eta_{Z, Y}$ are $\pi_{Y, X}$-related on $T_{Z} \cap T_{Y} \cap X$.

(d) If $Z<Y<X, \eta_{Y, X}$ is a sliding of $\eta_{Y, X} \mid T_{Z}(1) \cap T_{Y} \cap X$ along $\eta_{Z, X}$ restricted on $T_{Z} \cap T_{Y} \cap X$.

Let $\mathcal{S}_{X}$ be $\left\{Y_{1}, Y_{2}, \cdots, Y_{q}\right\}$ where the suffixes are chosen such that $\imath<\jmath$ if $\operatorname{dim} Y_{i}<\operatorname{dim} Y_{\jmath}$. For an element $x$ in $T_{Y_{1}} \cap X, S(x)$ denotes the set of strata $Y$ in $\mathcal{S}_{X}$ such that $x \in T_{Y}$. Let $(\mathrm{c})_{Z, Y}$ denote the condition (c) for the triple $Z<Y<X$.

Now, we assign for each $x \in X \cap T_{Y_{1}}$ a neighbourhood $U(x)$ on which a vector field satisfying the conditions (a), (c) and (d) can be constructed. If $\mathcal{S}(x)=\left\{Y_{1}\right\}$, there is a neighbourhood $U(x)$ such that $\mathcal{S}(y)=\mathcal{S}(x)$ for each $y \in U(x)$. We construct, in this case, a vector field satisfying the condition (a) on $U(x)$. If $\mathcal{S}(x) \neq\left\{Y_{1}\right\}$, then there exists $Y_{m} \in \mathcal{S}(x)$ to which each stratum in $\mathcal{S}(x)$ is incident. Then we assign a neighbourhood $U(x)$ such that $\mathcal{S}(y)=\mathcal{S}(x)$ for each $y \in U(x)$ and construct a vector field on $U(x)$ satisfying conditions (a) and $(\mathrm{c})_{Y_{1}, Y_{m}}$. Let $\left\{U_{\lambda}\right\}$ be a locally finite refinement of the covering $\{U(x)\}$. We construct $\eta_{Y_{1}, X}$ satisfying the conditions (a), (c) and (d) by means of a partition of unity associated to $\left\{U_{\lambda}\right\}$ and a certain normalization such that $\eta_{Y_{1}, X}$ satisfies the condition (b). Now we assume that $\eta_{Y_{j}, X}$ is defined for each $Y$, with $j<p$. Now we assign for each $x \in X \cap T_{Y_{p}}$ a neighbourhood on which a vector field satisfying the conditions (a), (c) and (d) can be constructed. Let $A$ be the set of those points $x \in X \cap T_{Y_{p}}$ such that $Y_{p}$ is incident to each stratum in $S(x)$. We assign $U(x)$ for each $x \in A$ such that $S(y)$ is equal to $\mathcal{S}(x)$ for each point $y$ in $U(x)$ and construct a vector field on $U(x)$ satisfying (a) and $(\mathrm{c})_{Y_{p}, Y_{m}}$. Here $Y_{m}$ denote the stratum in $S(x)$ to which each stratum in $\mathcal{S}(x)$ is incident. If $\mathcal{S}(x)$ contains a stratum which is incident to $Y_{p}$, we put $B$ the set of points $x \in T_{Y_{\imath_{1}}}(1) \cap \cdots \cap T_{Y_{\imath_{r}}}(1) \cap T_{Y_{p}} \cap T_{Y_{J_{1}}} \cap \cdots \cap T_{Y_{J_{m}}}$ with $\mathcal{S}(x)=\left\{Y_{\imath_{1}}, \cdots, Y_{\imath_{r}}, Y_{p}, Y_{\jmath_{1}}, \cdots, Y_{\jmath_{m}}\right\}$. For each point $x \in B$, we choose a neighbourhood $V(x)$ in $T_{Y_{\imath_{1}}}(1) \cap \cdots \cap T_{Y_{\imath_{r}}}(1) \cap T_{Y_{\jmath_{1}}} \cap \cdots \cap T_{Y_{\jmath_{m}}}$ such that each point $y$ in $V(x)$ satisfies the condition $\mathrm{A} 9$ and $\mathcal{S}(y)=\mathcal{S}(x)$ and construct a vector field $\eta_{x}$ on $V(x)$ satisfying (a) and (c) $Y_{p}, Y_{m}$. We slide each $\eta_{x}$ along 
$\eta_{Y_{\imath^{\prime}}, X}, \cdots, \eta_{Y_{\imath_{2}}, X}$ and $\eta_{Y_{\imath_{1}}, X}$ successively. Let $V^{*}(x)$ be the open set of all the elements of the form $\sigma_{t_{1}}^{Y_{\imath_{1}}{ }^{X}} \circ \ldots \circ \sigma_{t_{r}}^{Y_{\imath^{\prime}}{ }^{\prime} X}(y)$ with $y \in V(x)$. We extend $\eta_{x}$ over $V^{*}(x)$. It is clear that the family $\{U(x): x \in A\} \cup\left\{V^{*}(x): x \in B\right\}$ is an open covering of $T_{Y_{p}} \cap X$. Let $\left\{U_{\lambda}\right\}$ be a locally finite refinement of this covering. We construct $\eta_{Y_{p}, X}$ by means of a partition of unity associated to $\left\{U_{\lambda}\right\}$ and a certain normalization such that $\eta_{Y, X}$ satisfies $(\mathrm{a}) \sim(\mathrm{d})$. The required one parameter family $\left\{\sigma_{t}^{X}\right\}$ on $T_{Y} \cap X$ is generated by $\eta_{Y, X}$ for each $X \in \mathcal{S}$ and $Y \in \mathcal{S}_{X}$. By the construction, $\left\{\sigma_{t}^{X}\right\}$ satisfies the conditions $(1) \sim(5)$. This completes the proof of the lemma.

\section{$\S 3$. The proof of the theorem.}

Let $\{V, \mathcal{S}, \mathcal{I}\}$ be an $n$-dimensional stratified set satisfying $\mathrm{A} 10$ and $\mathrm{A} 11$. Let $V^{(k)}$ denote the union of all strata $X$ in $\mathcal{S}$ with $\operatorname{dim} X \leqq k$. Then, there is the number $k_{0}$ such that $V^{\left(k_{0}\right)}$ is the disjoint union of manifolds. Let $F^{\left(k_{0}\right)}$ be an embedding of $V^{\left(k_{0}\right)}$ into $R^{N}, \mathcal{S}\left(F^{\left(k_{0}\right)}\right)=\left\{F^{\left(k_{0}\right)}(X): X \subset V^{\left(k_{0}\right)}\right\}$ and $\Re$ the closed tubular neighbourhood system $\left\{\left(N(X), p_{X}, \tau_{X}\right): X \subset V^{\left(k_{0}\right)}\right\}$ for $\mathcal{S}\left(F^{\left(k_{0}\right)}\right)$ in $R^{N}$. We assume that there is a stratified realization $F^{(k)}$ of $V^{(k)}$ into $R^{N}$ and that $\mathcal{S}\left(F^{(k)}\right)=\left\{F^{(k)}(X): X \subset V^{(k)}\right\}$. Let $\Re$ be the closed tubular neighbourhood system $\left\{\left(N(X), p_{X}, \tau_{X}\right): X \subset V^{(k)}\right\}$ for $S\left(F^{(k)}\right)$ in $R^{N}$. As shown by Lemma 2, there exists a family of one parameter families of local transformations $\left\{\sigma_{t}^{X}\right\}_{X \in \mathcal{S}}$ for $\{V, \mathcal{S}, \mathscr{T}\}$ and there exists a family of one parameter families of local transformations $\left\{\beta_{t}^{X}\right\}_{X \in \mathcal{S}}$ for $\left\{F^{(k)}\left(V^{(k)}\right), \mathcal{S}\left(F^{(k)}\right)\right.$, $\left.\Re\right\}$. Let $X$ be a stratum in $\mathcal{S}$ with $\operatorname{dim} X=k+1$. Now we show that there exists an embedding $F_{Y}$ of $T_{Y} \cap X$ into $N(Y)$ for each $Y \in \mathcal{S}_{X}$, satisfying the following conditions:

(1) If $Z<Y$, then $F_{Z}=F_{Y}$ on $T_{Z} \cap T_{Y} \cap X$.

(2) $p_{Y} \circ F_{Y}=F^{(k)} \circ \pi_{Y}$.

(3) $\rho_{Y}(x)=\tau_{Y} \circ F_{Y}(x)$ for each $x$ in $T_{Y} \cap X$. We denote the elements of $\mathcal{S}_{X}$ by $Z_{1}, Z_{2}, \cdots, Z_{q}$ where we have $i<j$ whenever $Z_{\imath}<Z_{\jmath}$. Now we construct the embedding $F_{Z_{2}}$ of $T_{Z_{i}} \cap X$ to $N_{Z_{\imath}}$ successively. A sequence $\left(Y_{1}, \cdots, Y_{l}\right)$ of elements in $\mathcal{S}_{X}$ is said to be a chain of the length $l$ if $Y_{1}<Y_{2}<\cdots<Y_{l}$. For a chain $C=\left(Y_{1}, \cdots, Y_{l}\right)$, we apply Lemma 1 to the bundle $\pi_{Y_{1}}: T_{Y_{1}}(1) \cap \cdots$ $\cap T_{Y_{l}}(1) \cap X \rightarrow Y_{1} \cap T_{Y_{2}}(1) \cap \cdots \cap T_{Y_{l}}(1) \cap X$ to obtain the embedding $F_{C}{ }^{(1)}$ of $T_{Y_{1}}(1) \cap \cdots \cap T_{Y_{l}}(1) \cap X$ into $N_{Y_{1}}(1) \cap \cdots \cap N_{Y_{l}}(1)$. We can extend $F_{C}{ }^{(1)}$ to the embedding $F_{C}$ of $T_{Y_{1}} \cap \cdots \cap T_{Y_{l}} \cap X$ by $F_{C}\left(\sigma_{t 1}^{Y 1} \cdots \sigma_{t}^{Y}(x)\right)=\beta_{t 1}^{Y 1} \cdots \beta_{t}^{Y}\left(F_{C}{ }^{(1)}(x)\right)$. We define $F_{Z_{1}}$ on $T_{Z_{1}} \cap T_{Y_{1}} \cap \cdots \cap T_{Y_{k}} \cap X$ by $F_{C}$ for every $C=\left(Z_{1}, Y_{1}, \cdots, Y_{k}\right)$ with the maximum length. Now we define $F_{Z_{1}}$ on $T_{Z_{1}} \cap T_{Y_{1}} \cap \cdots \cap T_{Y_{\imath-1}} \cap T_{Y_{\imath+1}} \cap \cdots$ $\cap T_{Y_{k}} \cap X$. We apply Lemma 1 to the bundle $\pi_{Z_{1}}: T_{Z_{1}}(1) \cap T_{Y_{1}}(1) \cap \cdots \cap T_{Y_{2-1}}(1)$ $\cap T_{Y_{\imath+1}}(1) \cap \cdots \cap T_{Y_{k}}(1) \cap X \rightarrow Z_{1} \cap T_{Y_{1}}(1) \cap \cdots \cap T_{Y_{\imath-1}}(1) \cap T_{Y_{\imath+1}}(1) \cap \cdots T_{Y_{k}}(1) \cap X$ to extend the embedding $F_{Z_{1}}$ on the union of $T_{Z_{1}}(1) \cap T_{Y_{1}}(1) \cap \cdots \cap T_{Y_{2-1}}(1) \cap T_{Y}$ $\cap T_{Y_{\imath+1}}(1) \cap \cdots \cap T_{Y_{k}}(1) \cap X$ with $Y_{\imath-1}<Y<Y_{\imath+1}$ to $T_{Z_{1}}(1) \cap T_{Y_{1}}(1) \cap \cdots \cap T_{Y_{\imath-1}}(1)$ $\cap T_{Y_{\imath+1}}(1) \cap \cdots \cap T_{Y_{k}}(1) \cap X$. Then we define $F_{Z_{1}}$ on $T_{Z_{1}} \cap T_{Y_{1}} \cap \cdots \cap T_{Y_{\imath-1}} \cap$ 
$T_{Y_{\imath+1}} \cap \cdots \cap T_{Y_{k}} \cap X$ by means of the same procedure as in the extension of $F_{C}{ }^{(1)}$ to $F_{C}$. In this way, we can extend $F_{Z_{1}}$ step by step to reach finally the whole domain $T_{Z_{1}} \cap X$. Next we define $F_{Z_{2}}$. We define $F_{Z_{2}}$ on $T_{Z_{1}} \cap T_{Z_{2}} \cap X$ by $F_{Z_{1}}$. Let $\left(Z_{2}, Y_{1}, \cdots, Y_{m}\right)$ be a chain with the maximum length beginning with $Z_{2}$. We define $F_{Z_{2}}$ on $T_{Z_{2}} \cap T_{Y_{1}} \cap \cdots \cap T_{Y_{m}} \cap X$ by applying Lemma 1 to the bundle $\pi_{Z_{2}}: T_{Z_{2}}(1) \cap T_{Y_{1}}(1) \cap \cdots \cap T_{Y_{m}}(1) \cap X \rightarrow Z_{2} \cap T_{Y_{1}}(1) \cap \cdots \cap T_{Y_{m}}(1) \cap X$ to extend $F_{Z_{2}}$ on $T_{z_{1}} \cap T_{z_{2}}(1) \cap \cdots \cap X$ and by means of $\sigma_{t}$ and $\beta_{t}$. The extension of $F_{Z_{2}}$ to whole $T_{z_{2}} \cap X$ can be otained by the same procedure as in the case of $F_{Z_{1}}$. Similarly we can define the embedding $F_{Z_{\imath}}$ of $T_{Z_{i}} \cap X$. This completes the proof of the existence of $\left\{F_{Y}\right\}_{Y \in \mathcal{S}_{X}}$ and it is obvious by the construction that $F_{Y}$ satisfies the conditions (1) (3). We define the stratified realization $F$ by $F \mid V^{(k)}=F^{(k)}$ and $F=F_{Y}$ on $T_{Y}$ for each $Y \in \mathcal{S}_{X}$ with $\operatorname{dim} X$ $=k+1$. Let $\underline{X}$ denote the closure of the difference $X-\cup\left\{T_{Y}: Y \in \mathcal{S}_{X}\right\}$. The set $\underline{X}$ is a cornered manifold in the sense of J. Cerf ([5]). As shown in [5], we can extend $F$ to whole $X$ and we can choose the closed tubular neighbourhood $N(X)$ of $F(X)$ in $R^{N}$ with the projection $p_{X}$ and the tubular function $\tau_{X}$.

Now, we show that the image of the stratified realization satisfies the Whitney condition.

For any strata $X$ and $Y$ with $X>Y$ and each point $y$ in $Y$, by the definition of the tubular neighbourhood and the construction of the realization $F$, there exists a diffeomorphism $h$ from a neighbourhood $U$ of $F(y)$ in $R^{N}$ to $R^{N}$ such that $h(F(X) \cap U) \cong h(U) \times R^{N-c_{1}}$ and $h(F(Y) \cap U)=h(U) \times R^{N-c_{2}}$ where $c_{1}=$ codim $X$ and $c_{2}=\operatorname{codim} Y$. Since the pair $\left(R^{N-c_{1}}, R^{N-c_{2}}\right)$ with $c_{1}<c_{2}$ obviously satisfies the Whitney condition, the pair $(X, Y)$ also satisfies the same condition.

This completes the proof of the first part of the realization theorem. The remaining part of the theorem can be proved similarly.

\section{REFERENCES}

[1] H. Whitney, Elementary structures of real algebraic varieties, Ann. of Math. 66 (1957), pp. 545-556.

[2] J. MATHER, Notes on topological stability, Harvard University, preprint, (1970).

[3] R. Тном, Ensembles et morphisms stratifies, Bull. Amer. Math. Soc. 75 (1969), pp. $240-284$.

[4] R. Tном, Un lemma sur les applications differentiables, Bol. Soc. Math. Mexicana (1956), pp. 53-71.

[5] J. CERF, Topologie de certains espaces de plongements, Bull. Soc. Math. France 89 (1961), pp. 227-380. 